\title{
Matemáticas para Ingeniería: un Análisis del Funcionamiento Cognitivo en la Solución de un Problema de Cálculo en Dos Variables
}

\author{
Leopoldo Zúñiga-Silva \\ Instituto Tecnológico y de Estudios Superiores de Monterrey, Escuela de Ingeniería y Ciencias, \\ San Luis Potosí, México. (e-mail: Izs@itesm.mx)
}

Recibido Mar. 5, 2018; Aceptado Abr. 13, 2018; Versión final Jun. 20, 2018, Publicado Oct. 2018

\begin{abstract}
Resumen
El objetivo del estudio que se presenta fue realizar un análisis sobre el funcionamiento cognitivo de un grupo de estudiantes cuando abordaron la solución de un problema de cálculo diferencial de dos variables independientes. Es un estudio de carácter cualitativo basado en la observación sistemática y el análisis de conductas y respuestas de los alumnos en el proceso de solución del problema planteado. Los resultados de la investigación muestran cómo el funcionamiento cognitivo afecta la forma en que los alumnos comprenden e interpretan los elementos conceptuales y procedimentales en cada fase del proceso de solución. Se concluye que las funciones cognitivas analizadas se afectan por sesgos de pensamiento y por concepciones adquiridas durante la historia escolar de los alumnos.
\end{abstract}

\section{Mathematics for Engineering: An Analysis about Cognitive Functioning in the Solution of a Problem of Two Variables Calculus}

\begin{abstract}
The objective of the study presented in this paper was to analyze the cognitive functioning of a group of students when they confront the solution of a differential calculus problem on two independent variables. This is a qualitative study based on the systematic observation, conduct analysis, and student's answers in the solution's process of the given problem. This research shows how the cognitive functioning affects the way in which students understand and interpret the conceptual and operative elements in each phase of the solution process. The main conclusion was that the analyzed cognitive functions are affected by bias of thinking and by conceptions acquired during the student's scholar history.
\end{abstract}




\section{INTRODUCCIÓN}

En el ámbito de la investigación educativa en el área de las matemáticas se han realizado diversos estudios en relación a los elementos cognitivos involucrados en los procesos de aprendizaje (Roa-Fuentes y Parraguez, 2017; Benitez et al., 2016; D’Amore et al., 2015; Buitrago-Pulido, 2015; Vega, 2014; Radford y André, 2009; Ritter et al., 2007; Martínez y Argibay, 2007; Duval, 2006); Rodríguez, 2005). Sin embargo, tales investigaciones se enfocan en el estudio de elementos cognitivos propios del conocimiento matemático, como lo son las imágenes y las definiciones conceptuales (Dahl, 2017), o los esquemas mentales (Gagnier et al., 2017). También en los estilos cognitivos de aprendizaje (Kablan, 2016), las operaciones mentales (análisis, síntesis, analogías, etc.), y otros aspectos cognitivos involucrados en algunos actos de aprendizaje y sus implicaciones en la enseñanza (Tall y Katz, 2014), pero prácticamente no se ha realizado investigación sobre las funciones cognitivas que subyacen a tales elementos cognitivos, y a las propias operaciones mentales.

Es muy importante considerar esta situación, sobre todo porque en esos trabajos, y en particular los que se realizan con estudiantes universitarios, se toma como hecho que el funcionamiento cognitivo de los alumnos, es acorde con la supuesta madurez mental que deberían tener en función de su edad cronológica. Es menester, entonces, investigar sobre lo que sucede a nivel de funciones y procesos mentales respecto al aprendizaje, porque finalmente, aunque intervienen factores como hábitos, creencias, costumbres y otros factores sociales y culturales (y que, por su importancia, no pueden ser ignorados), éste sucede como resultado de procesos y operaciones mentales internas que dependen de un funcionamiento cognitivo individual (Bäuml, et al., 2017; Chang y Beilock, 2016).

La importancia de generar conocimiento en este ámbito es contar con información que posibilite impactar positivamente en el aprendizaje. Según Gutiérrez (2005, p.28), "La principal dificultad está en la necesidad que tenemos de conocer lo que pasa por la cabeza de los estudiantes cuando están envueltos en una actividad matemática, cuáles son sus procesos de razonamiento, cómo analizan y transforman la información que les llega del exterior, cuándo y cómo toman decisiones, etc. Todo ello para tratar de mejorar los procesos de enseñanza y aprendizaje".

Particularmente, interesa investigar lo que sucede a nivel cognitivo en un acto mental de aprendizaje en escenarios didácticos habituales. "Se entiende por sistema didáctico habitual aquel en que el profesor tiene el rol principal en el proceso de enseñanza-aprendizaje, mientras que los estudiantes asumen una actitud pasiva. El aprendizaje sucede principalmente por repetición, no por descubrimiento, lo cual conduce a un aprendizaje producto del énfasis en la mecanización del saber (esto no significa que el aprendizaje por repetición sea erróneo o inadecuado, sino que resulta insuficiente); además, la didáctica empleada está determinada por el discurso de los libros de texto." (Zúñiga, 2007, p. 148). Esto es, un ambiente educativo basado en explicaciones del profesor, realización de ejercicios y solución de problemas por parte de los alumnos, y utilizando libros de texto de uso común para la enseñanza y aprendizaje, en este caso del cálculo, orientados a la formación en ingeniería. De esta manera, se plantea la pregunta de investigación, ¿Qué sucede a nivel de funcionamiento cognitivo en alumnos de ingeniería cuando intentan resolver un problema matemático en un contexto habitual de enseñanza?

Para dar respuesta a esta pregunta, el objetivo de la investigación fue analizar el funcionamiento cognitivo de estudiantes universitarios en carreras profesionales de ingeniería, al enfrentarlos con el proceso de solución de un problema matemático de cálculo en dos variables independientes. En este trabajo se estudian los elementos cognitivos del aprendizaje cuando éste ocurre en escenarios didácticos con base en el planteamiento de problemas matemáticos relacionados al área de ingeniería.

\section{MARCO TEÓRICO}

Atendiendo el propósito de esta investigación, para lograr un análisis detallado del funcionamiento cognitivo de un grupo de estudiantes, se tomó como soporte la teoría de las funciones cognitivas de Feuerstein (1977). Esta teoría ofrece un catálogo de funciones cognitivas que los seres humanos ponen en juego al realizar actos mentales de aprendizaje. Las funciones cognitivas como actividades del sistema nervioso explican, en parte, la capacidad del individuo para servirse de la experiencia previa en su adaptación a nuevas situaciones (Feuerstein, 1979). Este referente es muy importante, considerando que en el presente trabajo se asume que los estudiantes construyen su propio aprendizaje en función del conocimiento previo que poseen (Mix et al., 2016; Tschentscher y Hauk, 2014).

Particularmente, esta investigación se llevó a cabo con base en el esbozo teórico implementado en un trabajo anterior propio (Zúñiga, 2007), en el cual se describen las características de cada fase (entrada, elaboración, salida) en el procesamiento de la información sobre las funciones cognitivas de Feuerstein, que aparecen en el acto mental de aprendizaje implicado en la resolución de un problema, y que se presentan a continuación. 
En la fase de entrada: la comprensión implica tener una percepción clara (1), tanto de los datos que se ofrecen en el enunciado, como del estado final o meta a la que se quiere llegar (los datos proporcionan una descripción completa del contexto del problema y de los parámetros bajo los cuales se debe operar). A su vez, para el logro de la percepción clara, es necesario que las funciones cognitivas de exploración sistemática de una situación de aprendizaje (2) y la de organización de la información (3), aparezcan en forma eficiente.

En la fase de elaboración: la resolución del problema implica la búsqueda de una vía de solución (una vía que conecte el estado inicial, con el estado meta), pero antes de esta búsqueda, es necesario que el sujeto sea capaz de percibir y entender con precisión el problema, lo cual implica que su función cognitiva de percepción y definición de un problema (4), aparezca en forma eficiente. Este es un momento crucial en el proceso, porque constituye el enlace entre la comprensión de la situación problemática y lo que es propiamente la resolución del problema. Se pueden tener dificultades en el desarrollo de la fase de elaboración, cuando no se define con precisión el problema en términos de la meta a la que se quiere llegar. La búsqueda de una vía de solución, implica la planificación de la conducta (5), así como la recuperación de esquemas en la memoria a largo plazo que involucran conocimientos matemáticos, y la cual, a su vez, requiere de una conducta comparativa (6).

El proceso de pensamiento para el uso, adecuación o modificación de esquemas previos en la construcción de las nuevas ideas, nociones o conceptos matemáticos, involucra al menos, la capacidad de pensamiento hipotético (7) y la conducta comparativa. La construcción de conocimiento requiere, para la codificación de la información correspondiente a las nuevas ideas, nociones y conceptos, de la función cognitiva de interiorización y representación mental (8), que es, de hecho, una de las funciones más importantes. En la fase de salida: la respuesta debe emitirse utilizando un lenguaje claro y preciso en función de la meta final del problema formulado; es decir, se debe observar una comunicación explícita (9) de tal respuesta. Se debe también observar capacidad para pensar y expresar la respuesta correcta al problema, así como para reflexionar antes de comunicarla, esto es, debe haber precisión y exactitud en la respuesta (10), y un control (11) en la emisión de la misma.

Las definiciones sobre las funciones cognitivas a las que se refiere este modelo teórico, son las siguientes: (1) Percepción clara: conocimiento exacto y preciso de la información. La disfunción cognitiva asociada, percepción borrosa, consiste en un proceso pobre e impreciso de los datos de la información. (2) Exploración sistemática de una situación de aprendizaje: es la capacidad para organizar y planificar la información. La disfunción de la exploración sistemática, es la impulsividad ante una situación de aprendizaje, consistente en una incapacidad para tratar la información de forma sistemática y planificada. (3) Organización de la información: capacidad para utilizar diferentes fuentes de información a la vez. (4) Percepción y definición de un problema: consiste en la habilidad para delimitar qué pide el problema, qué puntos hay que acotar, y cómo averiguarlos.

(5) Planificación de la conducta: capacidad para prever la meta que se quiere conseguir, utilizando la información adquirida previamente. (6) Conducta comparativa: consiste en la capacidad para realizar todo tipo de comparaciones y relacionar objetos y sucesos, anticipándose a la situación. La deficiencia en la conducta comparativa consiste en la incapacidad para establecer relaciones de semejanza y diferencia entre objetos y sucesos. (7) Pensamiento hipotético: capacidad para establecer hipótesis y comprobarlas aceptando o rechazando la hipótesis previamente establecida. (8) Interiorización y representación mental: capacidad para utilizar símbolos internos de representación. La falta o deficiencia de la interiorización, se manifiesta en la conducta demasiado concreta y sin generalización apropiada.

(9) Comunicación explícita: consiste en utilizar un lenguaje claro y preciso que responda al problema formulado en la tarea o problema. Esto supone un cierto nivel de comprensión por parte del sujeto. La disfunción correspondiente, es la comunicación egocéntrica. (10) Precisión y exactitud en las respuestas: capacidad para pensar y expresar la respuesta correcta a un problema o situación general de aprendizaje. (11) Control de las respuestas: consiste en la capacidad para reflexionar antes de emitir cualquier tipo de respuesta. El control y la autocorrección implican procesos meta-cognitivos.

Además, el estudio se complementa con soporte en la teoría de representaciones y sistemas representacionales (Goldin y Janvier, 1998; Goldin y Stheingold, 2001), y la teoría de esquemas cognitivos (Vergnaud, 1990; Dubinsky, 1991), ya muy conocidas en el ámbito de la investigación en didáctica de las matemáticas, pero también en la nueva tendencia de análisis cognitivo basada en el concepto de aprendizaje comunicacional o discursivo (Sfard, 2001).

Una representación se considera "una configuración cognitiva interna, individual, o un sistema complejo de tales configuraciones, inferida a partir de la conducta o la introspección, que describe algunos aspectos de los procesos del pensamiento matemático y la resolución de problemas. “, (Goldin y Janvier, 1998, p. 1). Un 
esquema cognitivo está formado por varios objetos y procesos relacionados a un concepto matemático particular (Dubinsky, 1991), y se concibe como "la organización invariante de la conducta para una cierta clase de situaciones" (Vergnaud, 1990, p.136). Por su parte, el aprendizaje comunicacional o discursivo, según Sfard (2001), se basa en el principio teórico de que "la comunicación no debería considerarse como una mera ayuda al pensamiento sino casi como equivalente al mismo pensamiento" (p. 13). Se considera que el aprendizaje ocurre como producto del dominio de un discurso, a través de la actividad y las relaciones interpersonales.

Este marco teórico permitió la realización de un análisis detallado de lo que sucede a nivel cognitivo respecto a la forma en que se manifiestan las funciones cognitivas de los estudiantes cuando resuelven un problema matemático, y su relación con representaciones, esquemas, y procesos de comunicación. Se describe a continuación la metodología y la forma en que se desarrollaron las actividades involucradas en esta investigación.

\section{METODOLOGÍA}

Se eligió un enfoque de carácter cualitativo (Hernández et al., 2014) de estudio de caso, basado en la observación sistemática y el análisis de conductas y respuestas de los alumnos en el proceso de solución del problema planteado. Este tipo de enfoque fue el más adecuado porque permitió interpretar y explicar el significado de los actos mentales observados, a la luz de las teorías utilizadas. La investigación se realizó mediante una actividad en el aula con un grupo de 16 estudiantes con edades entre 19 y 21 años, de un curso de cálculo de varias variables para estudiantes de ingeniería, en una institución de educación superior privada de México. La experiencia se realizó en un escenario de enseñanza común en el sistema habitual, esto es, mediante clases típicas donde el profesor asume el papel de guía, expone los temas que considera conveniente, y propone ejercicios y problemas a resolver a los estudiantes.

La recolección de datos se realizó mediante una guía de observación abierta (Valenzuela y Flores, 2013), con base en videograbaciones y la propia observación directa del investigador durante el desarrollo de las actividades, así como en el análisis de los documentos donde los estudiantes reportaron sus respuestas en el proceso de solución del problema. Por otra parte, el análisis se llevó a cabo mediante triangulación de la información obtenida (Hernández et al., 2014) de manera que se consideraron los datos recolectados, se realizaron las vinculaciones con la teoría, y se ofrecieron argumentos interpretativos propios, en cada fase del proceso, y en cada elemento cognitivo observado. Este recurso de análisis es muy importante en estudios de carácter cualitativo porque permite presentar un dato recolectado y confrontarlo con lo que dice la teoría al respecto, además de incorporar las interpretaciones y reflexiones del investigador, de manera que, en conjunto, se enriquecen las argumentaciones y se profundiza en el propio análisis de cada resultado.

El problema utilizado, extraído del texto de cálculo de Stewart (2006), fue el siguiente: Suponga que un científico tiene razones para creer que dos cantidades, $x$ e y se relacionan en forma lineal; es decir $y=m x+$ $b$, cuando menos de manera aproximada para algunos valores de $m$ y de $b$. El científico lleva a cabo un experimento y recopila datos en la forma de los puntos $\left(x_{1}, y_{1}\right),\left(x_{2}, y_{2}\right), \ldots\left(x_{n}, y_{n}\right), y$ los grafica. Los puntos no se encuentran exactamente en una recta, así que el científico desea determinar las constantes $m$ y b de modo que la recta $y=m x+b$ se "parezca" a los puntos tanto como sea posible (véase la Figura 1). Sea

$$
d_{i}=y_{i}-\left(m x_{i}+b\right)
$$

la desviación vertical del punto $(x, y)$ con respecto a la recta. El método de los mínimos cuadrados determina a $m$ y a b, de modo que minimiza

$$
\sum_{i=1}^{n} d_{i}^{2}
$$

que es la suma de los cuadrados de dichas desviaciones. Muestre que, de acuerdo con este método, la recta que más se "parece" se obtiene cuando:

$$
\begin{aligned}
& m \sum_{i=1}^{n} x_{i}+b n=\sum_{i=1}^{n} y_{i} \\
& m \sum_{i=1}^{n} x_{i}{ }^{2}+b \sum_{i=1}^{n} x_{i}=\sum_{i=1}^{n} x_{i} y_{i}
\end{aligned}
$$


Por lo tanto, la recta se determina resolviendo estas dos ecuaciones con las dos incógnitas m y b.

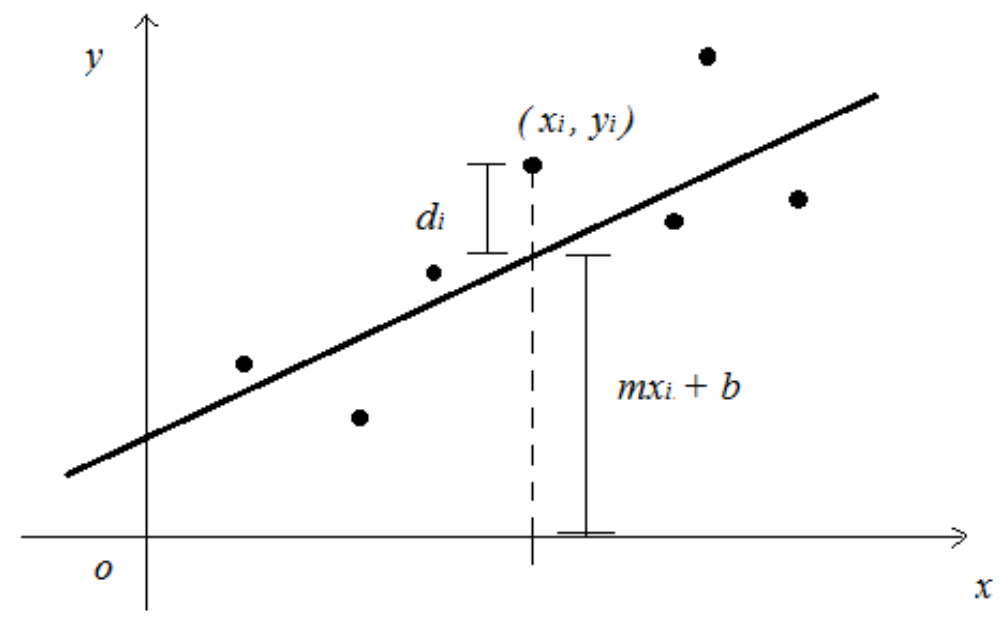

Fig. 1: Diferencia entre los puntos experimentales y la recta de ajuste buscada.

La experiencia se realizó de la siguiente forma: primero se presentó el problema a los estudiantes, distribuidos en cuatro equipos de cuatro integrantes, en una sesión de clase de 40 minutos, y se les pidió realizar lo que se indica en él. Después, al término de la sesión, se les pidió realizar lo siguiente en trabajo extra clase: Contesten y argumenten en la forma más amplia posible, las preguntas siguientes: ¿en qué consiste el método de los mínimos cuadrados?, ¿qué significa minimizar y qué conocimientos matemáticos se requieren para lograrlo?, ¿por qué?, ¿qué es la derivada parcial? Se les pidió entregar por escrito, en la clase siguiente, la solución al problema y respuestas a las preguntas. La finalidad, como se ha indicado, fue estudiar las funciones cognitivas de los estudiantes con base en el marco teórico, y explorar, además, sobre el estado de sus conocimientos matemáticos.

\section{ANÁLISIS DE RESULTADOS}

Los resultados se presentan de acuerdo a las partes y el orden en que se realizó la investigación: primero los correspondientes a la experiencia en el aula, y después los relacionados a la experiencia de los reportes escritos por equipo. Los análisis se realizaron atendiendo las características particulares del problema planteado, y de acuerdo con el siguiente mapa cognitivo:

Contenido: función de dos variables y manejo de derivadas parciales a nivel operativo. Modalidad: verbal, simbólica y gráfica. Operaciones: analogías, comparaciones y relaciones. Fases: en la fase de entrada, se requiere que el estudiante perciba que en el enunciado se describe en qué consiste la idea básica del método de mínimos cuadrados, y entonces lo que se pide es sólo minimizar la expresión (2) para llegar a las ecuaciones propuestas. En la fase de elaboración, primero es necesario que los alumnos, una vez que tienen claro lo que se pide hacer en el enunciado, sean capaces de acotar lo que se debe realizar a nivel operativo (por ejemplo, sustituir (1) en (2), para enseguida derivar parcialmente respecto a las variables $m$ y $b$ ) y, en su caso, averiguar lo que sea necesario. Deben tener la capacidad de recordar sus conocimientos sobre sumatorias e identificar que en la información aparece una función de dos variables.

Después, se debe recurrir a los conocimientos previos sobre la forma de determinar un mínimo, y emplear la simbología adecuada en el proceso de diferenciación. En la fase de salida, los estudiantes sólo deben verificar las ecuaciones que se proponen en el propio enunciado y hacerlo explícito verbalmente. Nivel de complejidad: medio-alto (los conocimientos y operaciones necesarias no requieren completamente de altos niveles de abstracción). Nivel de abstracción: medio-alto (intervienen varias nociones y símbolos de matemáticas avanzadas, tanto del curso en cuestión, como de otros precedentes). Nivel de eficiencia: medio. No es un nivel alto porque es considerable el grado de mecanización requerido.

\section{Análisis de la experiencia en el aula}

En la fase de entrada, se observaron dificultades para comprender lo que se plantea en la redacción del problema. A pesar de estar familiarizados con la simbología que se usa, se manifestaron conflictos en la comprensión. Estos conflictos van desde dudas sobre lo necesario para minimizar la sumatoria, y que se reflejan, por ejemplo, en preguntas como "¿lo que debemos hacer es derivar?”, hasta otros más profundos, por ejemplo, cuando algunos de los alumnos manifiestan que no entendían la explicación que se da sobre el método de mínimos cuadrados en el enunciado del problema, ni lo que se pide realizar en él. Comentaban 
cosas como "No entendemos nada, está muy largo", "Este problema está muy difícil,... dice muchas cosas", “¿cuál es el método de mínimos cuadrados?, eso no lo hemos visto, ¿usted nos lo va a explicar?”

Se puede inferir que la función de percepción clara se ve afectada en los estudiantes debido a la aparición de estimulación novedosa en la redacción del problema, por ejemplo, con el término "mínimos cuadrados"; también al nivel de complejidad, que el estudiante percibe en la simbología empleada, e incluso, a la amplitud del texto, lo que repercute en un conocimiento impreciso de los datos de la información. Esta situación está estrechamente relacionada con la pobre exploración sistemática observada. La impulsividad aparece en forma notable, por ejemplo, cuando los estudiantes advierten que se debe derivar, intentan hacerlo aún antes de tener claro cuál es la función a tratar y las variables involucradas (esto se presenta incluso después de trabajar extra clase, como se describe más adelante), al grado de que en dos de los equipos de trabajo empiezan a derivar parcialmente respecto a $x_{i}$ y $y_{i}$, sin advertir que las variables independientes en el problema, son $m$ y $b$. Estos alumnos ponen en juego un esquema mental (Dubinsky, 1991) basado en las representaciones simbólicas predominantes en la enseñanza habitual de las matemáticas desde los niveles básicos: los objetos matemáticos llamados variables o incógnitas, que más frecuentemente usan los profesores, son $x$ e $y$. Se emplean otras letras del alfabeto con mucha menor frecuencia.

Es importante señalar que el funcionamiento cognitivo se ve afectado también en forma considerable por otros dos aspectos notables: la falta de motivación y la consecuente falta de atención al abordar el problema. Aquí, es importante considerar que, en el aspecto motivacional, intervienen directamente sesgos de pensamiento, como los determinados por la creencia, o al menos duda constante, de que las matemáticas les serán poco útiles en su futuro ámbito profesional. Es decir, intervienen de manera decisiva factores de carácter sociocultural; por ejemplo, algunos estudiantes realizan lo que se les pide al resolver el problema, sólo por la motivación de obtener una calificación conveniente en la actividad, y no por lograr un verdadero aprendizaje. Aparecen comentarios como: "Está bien difícil, ... ¿cuánto vale del parcial?" (la pregunta alude a los puntos que vale la actividad de resolver el problema, respecto al periodo de evaluación parcial del curso). Al respecto, por ejemplo, Anaya-Durand y Anaya-Huertas (2010, p. 6), dicen que "Muchos de ellos (alumnos), quizá la mayoría, se encuentran solamente motivados por aprobar el curso, obteniendo una buena calificación y terminar la carrera lo antes posible, con un mínimo de esfuerzo y complicación."

Así, cuando se enfrentan al problema, predomina una actitud esquiva, tratan de evitar el enfrentarlo lo más posible, privilegiando aspectos superficiales del planteamiento, por encima de los datos relevantes. Por ejemplo, durante la sesión, al momento de iniciar la lectura del enunciado del problema, un alumno pregunta "¿Y esto para qué nos puede servir a nosotros? ..., aquí dice que le interesa a un científico...no es para un ingeniero". No se tiene en mente que un ingeniero pueda ser considerado un científico; quizá porque no se tiene claro qué es un científico. Otro alumno comenta que "Sí, este es un problema muy matemático, profe, mejor díganos cómo usar las fórmulas. Tiene ecuaciones muy difíciles."

Es evidente que estos conflictos en la fase de entrada no son consecuencia de factores asociados al desarrollo cognitivo de los alumnos, sino a "vicios" de pensamiento adquiridos a lo largo de su vida escolar. Situación que concuerda con lo señalado por Vergnaud (1990), cuando habla de su teoría de los campos conceptuales (aplicada en sus inicios a niños y adolescentes), en el sentido de que los elementos teóricos que la estructuran se refieren también a los procesos de aprendizaje del adulto, pero que estos últimos suceden bajo restricciones que son más del orden de los hábitos y de sesgos de pensamiento adquiridos, que relativos al desarrollo del aparato psíquico. Ideas que también se pueden soportar en la teoría de Piaget (1970) sobre las etapas de desarrollo intelectual, considerando que este trabajo se realizó con estudiantes de 19 a 21 años de edad.

Por otro lado, se observa que los alumnos tienen dificultades también con la función de organización de la información. Sin embargo, éstas no se deben a una incapacidad para realizarla, sino, más bien, a la incertidumbre en que ellos se encuentran como producto de las situaciones ya mencionadas, y a la presencia de estimulación novedosa. Por ejemplo, respecto al esquema (Dubinsky, 1991) que tienen los estudiantes de lo que es una función de dos variables (y cuáles son las variables) y a sus representaciones mentales (Goldin y Stheingold, 2001) sobre la noción de sumatoria. De su conocimiento previo, lo que surge y predomina es el significado sobre los símbolos. Asocian el símbolo $\sum$ a las series numéricas infinitas, lo cual implica el uso de la función cognitiva de conducta comparativa, sin embargo, pierden de vista la información en el enunciado del problema, y, en consecuencia, la necesidad o conveniencia del uso de este símbolo en el planteamiento. Aparecen comentarios como "sólo debemos derivar la serie, ¿no?”, y ¿cómo se deriva una serie?... ha... eso lo vimos en Mate 2". Esto, aludiendo al curso previo de cálculo integral en una variable y series numéricas infinitas.

Además, en esta primera fase de entrada, el proceso de comunicación en la actividad de aprendizaje, se ve afectado principalmente por dos condiciones desfavorables: el discurso plasmado desde la forma en que está 
redactado el problema, y la comunicación resultante de la interacción entre los propios alumnos, con base en esa redacción (en el enunciado del problema se explica en qué consiste el método de mínimos cuadrados, además de lo que se pide realizar). El aprendizaje es producto de las formas de comunicación y de las interacciones interpersonales entre los individuos (Sfard, 2001), pero en este proceso de inicio al abordar el problema planteado, no se cuenta con las condiciones adecuadas para establecer una comunicación efectiva.

En la fase de elaboración, como consecuencia de los conflictos observados en la fase de entrada, se ven afectadas las funciones de percepción y definición del problema, selección de información relevante, interiorización y representación mental, y la clasificación cognitiva. Las funciones de percepción y definición del problema, y la de selección de información relevante, están directamente relacionadas a las de percepción clara y exploración sistemática, y afectadas por ellas en términos de las observaciones indicadas anteriormente, desde la fase de inicio.

Respecto a la función cognitiva de interiorización y representación mental, se puede inferir su afectación en algunas acciones de los estudiantes, por ejemplo, cuando perciben que la función matemática involucrada es de dos variables independientes, les causa conflicto el que no aparezca en forma explícita la variable dependiente. Alguien comentó que "la función no tiene nombre". Además, aparecen dudas respecto a si la sumatoria es o no una función, y, por tanto, susceptible de diferenciación o no. Aparecen comentarios como "Las sumatorias no son funciones, son series, y las series no se pueden derivar, ¿o sí?", "Primero tenemos que desarrollar la sumatoria para poder derivarla, ¿no?”. Esto provoca que al momento de derivar no usen una simbología apropiada, por ejemplo, no indican qué es lo que van a derivar, sólo escriben el resultado.

La clasificación cognitiva es una función que a su vez depende de otras funciones, entre ellas las de percepción clara, y de conducta comparativa, ambas afectadas desde la fase de entrada. Pero, además, se presentan dificultades en ella debido a que los alumnos tienen deficiencias conceptuales respecto a las nociones matemáticas previas y necesarias, tales como sumatoria, función de dos variables, derivada parcial, valor mínimo de una función de dos variables, etc. Sus esquemas y representaciones (Goldin y Stheingold, 2001) relacionadas a estas nociones, resultan insuficientes o inadecuados para soportar eficientemente el tránsito entre sus conocimientos previos, y los nuevos recursos conceptuales y procedimentales necesarios para entender y ofrecer una solución al problema.

Finalmente, en la fase de salida, sólo se observaron conflictos en la función de comunicación explícita y en la de precisión y exactitud de la respuesta. Algunos estudiantes llegan a las ecuaciones indicadas, pero no escriben la respuesta en forma explícita. Se puede decir, de acuerdo a la teoría, que presentan una comunicación egocéntrica: no consideran necesario mayor explicación sobre la solución, asumen que cualquier persona que vea su trabajo lo comprenderá bien en los términos que ellos lo presentan.

\section{Análisis sobre los reportes escritos}

Algunos de los conflictos y deficiencias observadas en la solución del problema prevalecen aún después del trabajo extra clase que realizaron los estudiantes. Respecto a la primera pregunta: ningún equipo contesta (recordemos que la pregunta era, ¿en qué consiste el método de los mínimos cuadrados?) haciendo referencia explícita al enunciado del problema. Todos los estudiantes se dieron a la tarea de investigar al respecto y presentan en su reporte resúmenes, información parcial, e incluso copias exactas de libros donde se trata el método. Esta es una situación esperada puesto que, de acuerdo a lo observado durante la sesión en el aula, la información proporcionada en el enunciado del problema, resultó no ser suficiente para la comprensión del método.

Respecto a la segunda pregunta, ¿Qué significa minimizar y qué conocimientos matemáticos se requieren para lograrlo?, ¿por qué?, se observó que no interiorizan adecuadamente debido a factores conceptuales sobre sus conocimientos previos, por ejemplo, uno de los equipos, como parte de su respuesta a la pregunta, escribe: "Minimizar significa reducir a su mínimo volumen, en este caso específico se refiere a buscar el mínimo de una función, es decir hallar el límite inferior al que puede reducirse." Parece ser que la concepción de minimizar en estos estudiantes gira en torno a la noción de volumen. Para ellos, minimizar significa buscar el mínimo de una función o determinar el límite inferior al que puede reducirse (el volumen). Esta concepción se hace más evidente cuando en este mismo equipo escriben que: "Para encontrar dicho mínimo se utiliza la derivada ya que es el único procedimiento por el cual se encuentran mínimos ya que al integrar se encuentra el máximo pues es esta se suman las diferenciales". Esto indica que el conocimiento que los alumnos tienen respecto a derivar e integrar, quedó codificado en su memoria a largo plazo como quitar y agregar, respectivamente, y es eso lo que recuperan de los conceptos implicados al pensar sobre la pregunta. Derivar, entonces, significaría reducir al mínimo un volumen (restar), e integrar, por el contrario, permitiría encontrar el máximo (sumar). 
En esta situación aparece un esquema muy particular basado en la noción de volumen, desafortunadamente, el diseño de esta investigación no permitió contar con elementos para analizar las causas que le dieron origen. Se podría suponer que se debe a alguna exposición particular de estos alumnos a una experiencia previa de aprendizaje, especifica, donde la noción de volumen era un elemento central. Lo que sí se pudo observar, es que en las condiciones que se dieron en este estudio, los alumnos tuvieron una afectación cognitiva tal que, en ese momento particular de la actividad, no pudieron percatarse de que un "volumen mínimo" no tendría sentido.

Por otra parte, respecto a la idea del procedimiento analítico para determinar el mínimo escriben que “...a partir de las raíces de la derivada podemos ver en la gráfica los puntos críticos que en ella existen, entonces encontramos el mínimo relativo o absoluto y por ende se encuentra el error mínimo posible. Se hallará la derivada parcial de primero orden, en este caso con dos variables, se derivará con respecto de ambas variables, se iguala el resultado a cero con el fin de encontrar las raíces y así los puntos críticos y se resuelve para encontrar los valores". Se puede observar aquí que el elemento central en estas ideas queda constituido por las raíces de la derivada.

Los estudiantes asumen que la función derivada tiene raíces y entonces sólo es cuestión de encontrarlas. No es posible determinar con precisión si los alumnos tienen claro que la función del problema, por sus características, siempre tiene un mínimo, y por eso el derivar parcialmente, igualar a cero y resolver el sistema, funciona siempre. Sus argumentos pueden deberse a esto, pero también pueden ser consecuencia de que el algoritmo derivar-igualar a cero-resolver es el esquema que ha quedado en su memoria y que consideran aplicable a toda función de dos variables, sin necesidad de considerar otras posibilidades de valores extremos (por ejemplo, el mínimo en el "pico" de un cono que "abre" hacia arriba). Este esquema aparece entonces como un invariante operatorio (Verganud, 1990) producto de las experiencias de aprendizaje que han vivido los alumnos en sus cursos previos de cálculo de una variable, y que ponen en juego cada vez que requieren calcular un punto crítico.

En relación a la tercera pregunta: el resultado más importante aquí fue que todos los equipos inician su respuesta a esta pregunta (¿qué es la derivada parcial?) describiendo la forma de calcular una derivada parcial. No contestan de entrada qué es, sino, cómo calcularla operativamente. Posteriormente mencionan la definición en términos de límites (copiada de libros) y sólo tres de los equipos, al final de su respuesta, presentan la interpretación geométrica. Evidentemente, del conocimiento que tienen los alumnos sobre la derivada parcial, la parte operativa es la que predomina. La manipulación de objetos matemáticos está primero que la comprensión de elementos conceptuales. En sus esquemas tiene prioridad el registro algebraico, primero aparece la forma de determinar una derivada (la idea de que, al derivar una función de dos variables respecto a una de ellas, la otra se trata como constante), y después las definiciones e interpretaciones geométricas. Nuevamente, se observa una condición de sesgo de pensamiento (Vergnaud, 1990) que no se debe a deficiencias particulares en sus funciones cognitivas, sino a representaciones mentales fuertemente vinculadas a la idea de que lo más importante en matemáticas es el trabajo algebraico.

Se aclara que estas últimas observaciones y reflexiones (en los reportes escritos) son un intento de explorar las causas de los aspectos conceptuales que intervienen en los procesos cognitivos al abordar un problema, pero no se pretendió realizar un estudio exhaustivo en este sentido (esto, por sí mismo, requeriría un estudio aparte). Lo que sí se puede decir en relación al objetivo de esta investigación, es que estas concepciones previas y sesgos de pensamiento de los estudiantes, influyen decisivamente en su funcionamiento cognitivo, en particular sobre las funciones de interiorización y representación mental y la de precisión y exactitud en la respuesta.

\section{CONCLUSIONES}

El marco teórico utilizado permitió realizar el análisis cognitivo planteado. Dentro de los principales resultados se encontró que las funciones cognitivas analizadas se vieron afectadas por los sesgos de pensamiento y las concepciones adquiridas durante la historia escolar de los alumnos. Los conflictos observados no fueron producto de incapacidades atribuibles a factores propios del desarrollo cognitivo, ni a un estado de disfunción permanente, sino al efecto de las disfunciones locales que aparecieron en actos mentales específicos y que fueron provocadas, principalmente, por la forma en que se abordó y desarrolló la experiencia de aprendizaje.

De esta forma, las disfunciones observadas sucedieron como consecuencia de la exposición de los estudiantes al escenario habitual de enseñanza utilizado, lo cual provocó que aparecieran sesgos de pensamiento desarrollados en ellos como consecuencia de su exposición al sistema educativo tradicional. El diseño didáctico utilizado en esta investigación, propició, en ciertos momentos del proceso de solución, aprendizajes erróneos, o por lo menos, incompletos o deficientes, que se convirtieron en un obstáculo para un funcionamiento cognitivo adecuado. 
El problema que se utilizó es de un tipo común en libros de texto de cálculo. Está diseñado con un discurso didáctico basado en la enseñanza habitual (Zúñiga, 2007), que, de acuerdo a los resultados de esta investigación, no favorece el aprendizaje que se pretende en los alumnos. En este caso particular, debido principalmente a que el enunciado incluye la explicación del propio método de mínimos cuadrados, lo cual tiene como consecuencia que, además de tener una redacción muy extensa, aparece demasiada información simbólica, lo cual propició la aparición de los conflictos cognitivos observados.

Finalmente, se puede inferir también que la madurez mental no garantiza la eficiencia de las funciones cognitivas en un acto mental de aprendizaje. Esto debido, sobre todo, a que ese acto mental es afectado tanto por los conocimientos previos de los estudiantes, como por el sistema de creencias y sesgos de pensamiento asociados a factores didácticos y socioculturales respecto al conocimiento que se aborda; en este caso, el conocimiento matemático.

\section{REFERENCIAS}

Anaya-Durand, A. y C. Anaya-Huertas ¿Motivar para aprobar o para aprender? Estrategias de motivación del aprendizaje para los estudiantes, Tecnología, Ciencia, Educación 2010, 25 (1), 5-14 (2010)

Bäuml, J.G., C. Meng y otros nueve autores, The association of children's mathematic abilities with both adults' cognitive abilities and intrinsic fronto-parietal networks is altered in reterm-born individuals, DOI: 10.1007/s00429-016-1247-4, Brain Structure and Function, 222 (2), 799-812 (2017)

Benitez, A.A., H. Benitez y M.L. García, La argumentación sustancial. Una experiencia con estudiantes de Nivel Medio Superior en clases de matemáticas, Educación Matemática, 28(3), 175-216 (2016)

Buitrago-Pulido, R.D., Incidencia de la realidad aumentada sobre el estilo cognitivo: caso para el estudio de las matemáticas, DOI: 10.5294/edu.2015.18.1.2, Educ. Educ. 18(1), 27-41 (2015)

Chang, H. y S. L. Beilock, The math anxiety-math performance link and its relation to individual and environmental factors: A review of current behavioral and psychophysiological research, DOI: 10.1016/j.cobeha.2016.04.011, Current Opinion in Behavioral Sciences, 10, 33-38 (2016)

Dahl, B., First-Year Non-STEM Majors' Use of Definitions to Solve Calculus Tasks: Benefits of Using concept Image over Concept Definition? DOI: 10.1007/s10763-016-9751-9, International Journal of Science and Mathematics Education, 15 (7), 1303-1322 (2017)

D’Amore, B., M. Fandiño, M. lori y M. Matteuzzi, Análisis de los antecedentes histórico-filosóficos de la "paradoja cognitiva de Duval", DOI: 10.12802/relime.13.1822, Revista Latinoamericana de Investigación en Matemática Educativa, 18 (2), 177-212 (2015)

Dubinsky, E., Reflective abstraction in advanced mathematical thinking, Advanced Mathematical Thinking, Dordrecht: Kluwer, A. P. 95-123 (1991)

Duval, R., A Cognitive Analysis of Problems of Comprehension in a Learning of Mathematics, Educational Studies in Mathematics, 61, 103-131 (2006)

Feuerstein, R., Mediated Learning Experience: A theoretical basis for cognitive human modificability during adolescence. In Mittler P., Research to practice in mental functions, University Park Press, Vol. 2, Baltimore, EU (1977)

Feuerstein, R., The Dynamic Assessment of Retarded Performes: The Learning Potential Assessment Device, Theory, Instruments and Techniques, University Park Press, Baltimore, EU, (1979)

Gagnier, K.M., K. Atit, C.J. Ormand y T. F. Shipley, Comprehending 3D Diagrams: Sketching to Support Spatial Reasoning, DOI: 10.1111/tops.12233, Topics in Cognitive Science, 9 (4), 883-901 (2017)

Goldin, G. y C. Janvier, Representation and the psychology of mathematics education, Journal of Mathematics Behaviour, 17(1), 1-4 (1998)

Goldin, G. y Stheingold, System of representations and the development of mathematical concepts, The roles of representation in school mathematics, NCTM, 1-23 (2001)

Gutiérrez, A., Aspectos metodológicos de la investigación sobre aprendizaje de la demostración mediante exploraciones con software de geometría dinámica, Noveno Simposio de la Sociedad Española de Educación Matemática, 27-44, Córdova, España, 7 al 10 de septiembre (2005)

Hernández, R., C. Fernández y P. Baptista, Metodología de la Investigación, 6aㅡ Ed., Mc Graw Hill, México, D.F. (2014)

Kablan, Z., The effect of manipulatives on mathematics achievement across different learning styles, DOI: 10.1080/01443410.2014.946889, Educational Psychology, 36 (2), 277-296 (2016)

Martínez, J. y P. Argibay, El aprendizaje de las matemáticas y el cerebro, Ciencia Hoy, 17(99), 46-51 (2007)

Mix, K. S., S. C. Levine, Y. L. Cheng, C. Young, D. Z. Hambrick, R. Ping y S. Konstantopoulos, Separate but correlated: The latent structure of space and mathematics across development, DOI: 10.1037/xge0000182, Journal of Experimental Psychology: General, 145 (9), 1206-1227 (2016) 
Piaget, J., Intellectual Evolution from Adolescence to Adulthood, Human Develop, 15, 1-12 (1972)

Radford, L. y M. André, Cerebro, cognición y matemáticas, Revista Latinoamericana de Investigación en Matemática Educativa, 12(2), 215-250 (2009)

Ritter, S., J. R. Anderson, K. R. Koedinger y A. Corbett, Cognitive tutor: applied research in mathematics education, Psychonomic bulletin \& review, 14 (2), 249-255 (2007)

Roa-Fuentes, S. y M. Parraguez, Estructuras Mentales que Modelan el Aprendizaje de un Teorema del Álgebra Lineal: Un Estudio de Casos en el Contexto Universitario, DOI: 10.4067/S0718-50062017000400003, Formación Universitaria, 10(4), 15-32 (2017)

Rodríguez, I., La resolución de problemas y el pensamiento matemático divergente, Revista Ciencias, 2 (14), $72-97$ (2005)

Sfard, A., There is more to discourse than meets the ears: Looking at thinking as communicating to learn more about mathematical learning, Educational Studies in Mathematics, 46, 13-57 (2001)

Stewart, J., Cálculo, conceptos y contextos, $3^{\text {ra }}$ Ed., International Thomson Editores, Distrito Federal, México (2006)

Tall, D. y M. Katz, A cognitive analysis of Cauchy's conceptions of function, continuity, limit and infinitesimal, with implications for teaching the calculus, DOI: 10.1007/s10649-014-9531-9, Educational Studies in Mathematics, 86, 97124 (2014)

Tschentscher, N. y O. Hauk, How are things adding up? Neural differences between arithmetic operations are due to general problem solving strategies, DOI: 10.1016/j.neuroimage.2014.01.061, Neurolmage, 92, 369-380 (2014)

Valenzuela, J. y M. Flores, Fundamentos de investigación educativa, Vol. 2: El proceso de investigación educativa, Digital, Monterrey, NL, México (2013)

Vega, M. A., J. Carrillo y J. Soto, Análisis según el Modelo Cognitivo APOS* del Aprendizaje Construido del Concepto de la Derivada, DOI: 10.1590/1980-4415v28n48a20, Bolema: Boletín de Educación Matemática, 28(48), 403-429 (2014)

Vergnaud, G., La théorie des champs conceptuels, Recherches en Didáctique des Mathématiques, 10(2), 133-170 (1990)

Zúñiga, L., El cálculo en carreras de ingeniería: un estudio cognitivo, Revista Latinoamericana de Investigación en Matemática Educativa, 10(1), 145-175 (2007) 